\title{
Bio-inspired Collision Detector with Enhanced Selectivity for Ground Robotic Vision System
}

Qinbing Fu
10230460@students.lincoln.ac.uk

Shigang Yue

syue@lincoln.ac.uk

Cheng $\mathrm{Hu}$

chu@lincoln.ac.uk

\author{
Computational Intelligence Laboratory \\ Department of Computer Science \\ University of Lincoln \\ Lincoln, UK
}

There are many ways of building collisiondetecting systems. In this paper, we propose a bio-inspired collision detector based on the juvenile locust vision pathway (Fig.1). Two type$\mathrm{s}$ of motion detectors, LGMD1 and LGMD2 have been identified in locusts' visual system [1]. Compared to LGMD1, LGMD2 matures early in juvenile locusts which mainly live on the ground whereas already represent evasive responses to swooping predators. An importan$\mathrm{t}$ feature is its looming sense is only for lightto-dark luminance change. It is able to detec$\mathrm{t}$ dark looming objects embedded in the bright background selectively whilst not responding to light objects against the dark background. There are two defects in LGMD1 modeling works (e.g. [2]): first, the approaching and receding stimulus are not properly distinguished in depth; second, the translating stimulus regularly leads to collision mis-detection. The revealed neural characteristics of LGMD2 make it ideal to handle those defects for ground vision-based platforms. Compared to some state-of-the-art collision detectors, the proposed bio-inspired computational model can cope with unpredictable environments without using specific object recognition algorithms. It detects potential collision via reacting to expansion of the object edges, rather than the strategy of recognizing the target or analyzing the scene.

The core of this framework is a biophysical architecture of ON and OFF visual pathways, which underlies motion detection circuit, and reveals the fundamental principle of splitting visual signals into parallel channels encoding brightness increments $(\mathrm{ON})$ and decrements $(\mathrm{OFF})$ as illustrated in Eq.1. Moreover, in LGMD2 modeling work, we put forth a bias in ON pathway to achieve its specific collision selectivity.

The proposed framework was set up in a vision-based ground miniature robot and tested against systematic and comparative real-time ex- periments. Compared to other computer vision techniques, this neural system performs quickly and robustly in the very limited hardware. The experimental results also demonstrate two main contributions: first, the collision selectivity to dark objects against bright background is enhanced which makes it ideal for ground mobile robots; second, the selectivity to approaching objects versus translation has been shaped which is expected for a practical collision-detecting system.

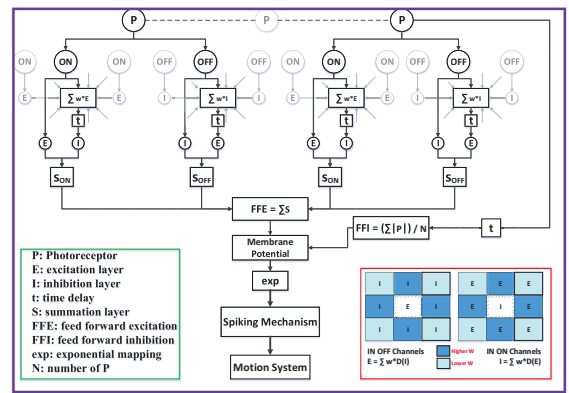

Figure 1: The schematic overview of LGMD2 vision system: Model notations are illustrated in green-box. The convolution illustrations are in red-box.

$$
\begin{aligned}
& P_{x, y}^{O N}(t)=\left(P_{x, y}(t)+\left|P_{x, y}(t)\right|\right) / 2, \\
& P_{x, y}^{O F F}(t)=\left|\left(P_{x, y}(t)-\left|P_{x, y}(t)\right|\right)\right| / 2
\end{aligned}
$$

[1] P. J. Simmons and F. C. Rind. Responses to object approach by a wide field visual neurone, the lgmd 2 of the locust: Characterization and image cues. J Comp Physiol A, 180:203-214, 1997.

[2] S. Yue and F. C. Rind. Collision detection in complex dynamic scenes using a lgmd based visual neural network with feature enhancement. IEEE Trans. Neural Netw., 17(3):705-716, 2006. 